Optical Society of America. Hitoshi Fujii and Silverio P. Almeida, "Coherent spatial filtering with simulated input," Appl. Opt. 18, 1659-1662 (1979). doi: 10.1364/ao.18.001659

\title{
Coherent spatial filtering with simulated input
}

\author{
Hitoshi Fujii and Silverio P. Almeida
}

\begin{abstract}
A disadvantage of a well-tuned matched filter is partly overcome in recognizing biological species, i.e., algae. A simplified input pattern is prepared which simulates a given species. The matched spatial filter for this pattern can identify various diatoms in the species with considerably less size and orientation dependence. In addition, the filter still retains a high degree of pattern discrimination against other species.
\end{abstract}

\section{Introduction}

Matched spatial filters (MSF) play an important role in pattern recognition optical processors. ${ }^{1-5}$ Highly similar patterns can be discriminated by tuning in the desired spatial frequencies via the proper ratio of the signal to reference beams and the correct exposure time. However, one disadvantage to well-tuned filters is their high sensitivity to small rotation and size differences for a given input. 6 The higher the spatial frequencies recorded on a filter the more sensitive is the filter. This problem is more serious in the application of MSF to biological specimens, such as diatoms (algae) treated here. ${ }^{2}$

Needless to say, any biological input patterns are never entirely the same. For example, diatoms belonging to even a same species are quite similar in shape, but different in size and their stria patterns. ${ }^{7}$

Although one well-tuned MSF can pick up some diatoms of similar shape, size, and orientation, it covers only a very small part in the ensemble of input diatoms. The recognition efficiency has been too poor to construct any practical systems.

To obtain higher efficiency and solve a part of this problem, this paper studies the characteristics of MSF made by a simplified pattern simulating a given input species. The simplified MSF provides a way of considerably lessening the sensitivity to input orientations and size variations. In addition the filter still retains its high degree of discrimination. The optical processor used to obtain the results presented here has been described in previous papers. ${ }^{2,8,9}$

The authors are with Virginia Polytechnic Institute and State University, Physics Department, Blacksburg, Virginia 24061.

Received 1 November 1978.

0003-6935/79/101659-04\$00.50/0.

(C) 1979 Optical Society of America.

\section{Preparation of Input}

In a hybrid optical processor, which uses MSF for pattern recognition, one normally prepares a Fourier transform hologram of a given input pattern and correlates it against various unknown patterns in order to see the number of times, if any, that it exists. A filter can be highly tuned by recording higher spatial frequencies of the original input, which in turn results in a higher rate of discrimination against the other input patterns. As pointed out earlier such filters may be useless in the pattern recognition of biological specimens. In any event the rotation and size limitations for a normal filter are about $\pm 5^{\circ}$ and $4 \%$ size variation, ${ }^{8}$ while diatoms in the same species can have any orientation and $50 \%$ size variation in an actual slide input. Therefore, it is often advantageous to sacrifice a high rate of discrimination against the diatoms in the other species in favor of a higher recognizing rate for those in their own species. One way to achieve this is to alter the original input used to make the filter. However, it is not obvious as to what these input changes should be.

As a preliminary study, we modified the original input pattern into simpler forms shown in Figs. 1(a)-1(c). Figure 1(a) shows a diatom, which is normally taken to be our input. Its structure is seen to contain both low spatial frequencies (edge contours) as well as higher components (interior grating like structure). Figure 1 (b) is a first approximation to this input in which the high frequencies are removed; (c) is one of the simplest forms characterizing the original input (a). The horseshoelike pattern was carefully drawn so as to overlap partly to various input patterns. Three different Fraunhofer holograms were made and used in MSF experiments against another set of patterns.

The input patterns to be examined are shown in Fig. 2. In the first row, there are thirteen diatoms in the same species whose length varies down to $50 \%$. It is also noticed that interior structure (stria) are different from each other just as their individuality. However, each pattern is seen to have similar features in shape, such 


\section{㽬}

(a)

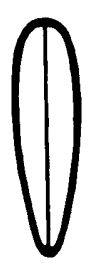

(b) $\cap$

(c)

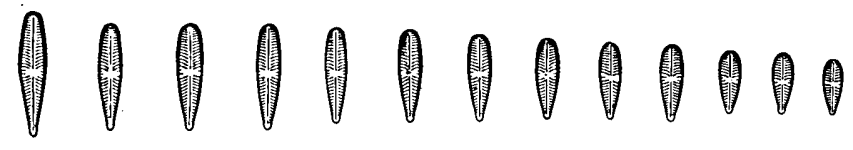

Fig. 1. (a) The original diatom specimen photographed through a phase contrast microscope. Parts (b) and (c) each show a simulated input for (a). In (c) the horseshoe shaped figure contains only low spatial frequencies.

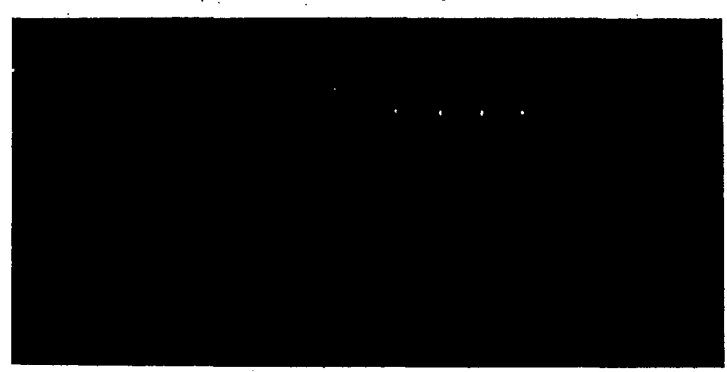

(a)

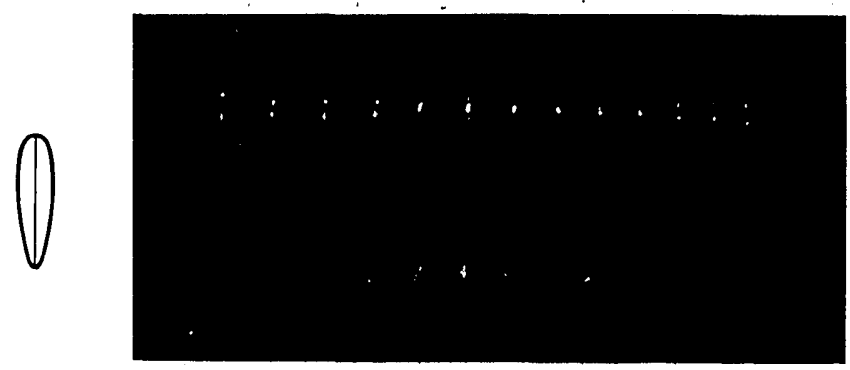

(b)

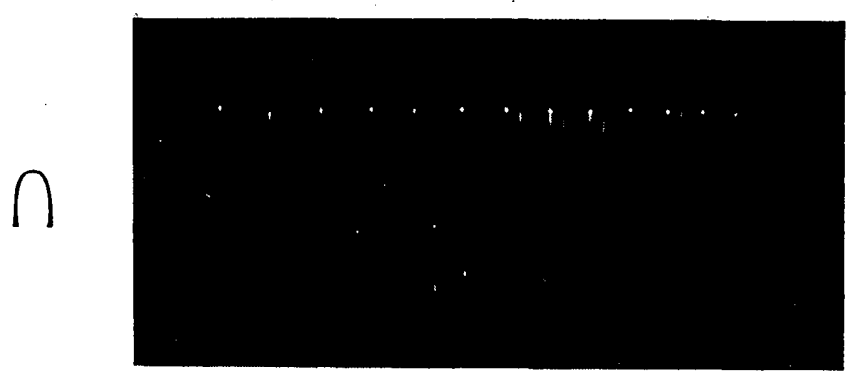

(c)

Fig. 3. (a) The original input pattern (left) used for MSF and its correlation signals (right) against the input shown in Fig. 2. The location of the exact autocorrelation signal produced by the original input is the second dot from the right. (b) The first approximated pattern and the correlation signals against the same input. (c) The horseshoe pattern simulating the diatom in (a) and its cross-correlation signals against the input shown in Fig. 2.

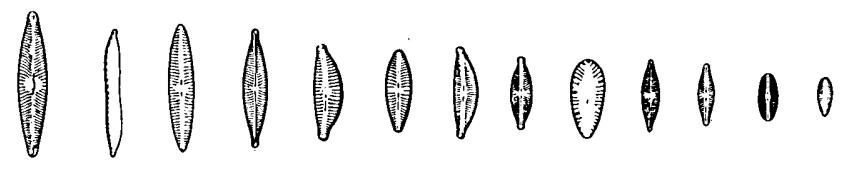

Fig. 2. The top row of thirteen diatoms shows the same species but varying in size by as much as $50 \%$. The bottom row contains thirteen diatoms mostly similar to the top row but which are different species.

as horseshoelike top and wedged bottom half. The second row shows thirteen other diatom species to be discriminated from the upper row diatoms, which are, on the contrary, to be identified.

\section{Results and Discussion}

Figures 3(a)-3(c) show the results of cross-correlation signals obtained in the output plane of a MSF experimental system. ${ }^{2}$ The left side of each figure is the input pattern from which the filter was made. In Fig. 3(a), since the filter was made with the fifth diatom from the right, a brightest spot of the exact autocorrelation appears at the same position. It is seen that this welltuned filter can identify only a few of the other similar sized diatoms.

Figure 3(b) are the correlation signals of the contoured pattern against the test input. All diatoms in the first row are recognized with bright spots, while a few false correlation signals did occur in the second row. However, some correlation signals in the upper row are seen to have a double peak. This is because the top and bottom contours of the simulated pattern can correlate with different parts of the inputs. Such double peaks may result in miscounting and complicate the analysis. This problem is overcome by making a filter with the simplest horseshoelike pattern shown in Fig. 1(c).

Figure 3(c) shows the new filter's success in identifying size differences for the same species as well as its high degree of discrimination against different species except one false signal at the sixth from the left. Since this filter is made by a simple shape, each correlation pattern has higher side lobes compared with the central peak.

Next the filter was tested for rotational sensitivity against the same 26 diatoms shown in Fig. 2. The results are presented in Figs. 4(a) and 4(b), for $\pm 30^{\circ}$ of rotation. It is seen that this filter retains its recognition ability as well as being considerably less sensitive to rotations in the input plane. For this species, multiple exposed filters with about seven different angles may cover the entire rotation of the input. 


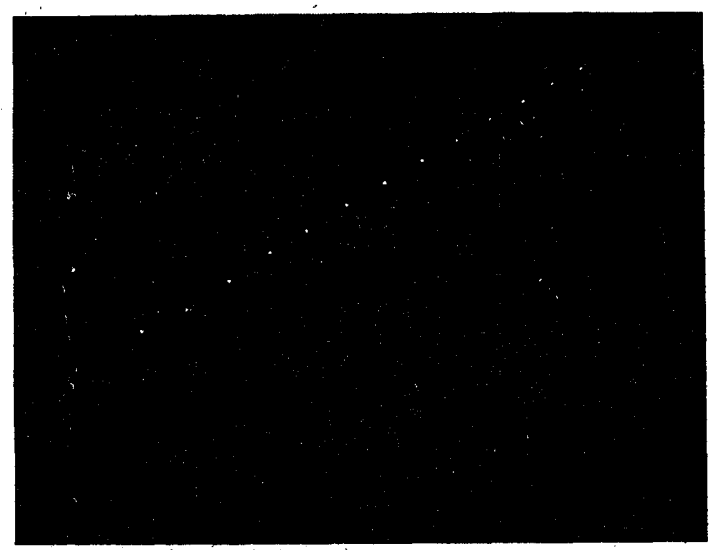

(a)

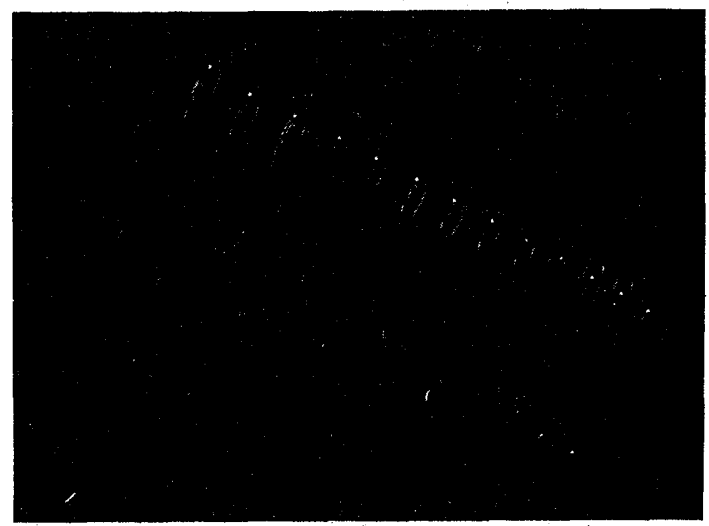

(b)

Fig. 4. Cross-correlation signals obtained with the horseshoe filter and rotating Fig. 2 input $\left( \pm 30^{\circ}\right)$.

Figure 5 shows the actual peak heights of correlation signals shown in Figs. 3 and 4, arranged in the matrix form. The scale changes to double from the fifth column as indicated with the unit mark $I$. The input pattern was rotated to $45^{\circ}$ with the increment of $15^{\circ}$. With a proper threshold level, most of the diatoms in this species (upper row in Fig. 2) are expected to be identified while others are discriminated.

Finally we show the spectral distribution of the simulated input and compare it against that of the original species. Figure 6(a) is the power spectrum of the horseshoelike input shown in Fig. 1(c). The pattern seems to consist of two symmetric sets of parabolas having stronger intensity at their vertices. On the other hand, Fig. 6(b) is the average power spectrum of an input consisting of $N$-randomly distributed diatoms in the original species. ${ }^{7}$ As discussed in the Ref. 7 the pattern indicates the basic spectral distribution, which characterizes the similar feature in the given set of input patterns. It is seen that these two power spectra are quite similar in the lower spatial frequencies and can overlap together in some different orientation.
There are some other simplified patterns simulating the original species, such as a wedged bottom or a pair of symmetric arcs. These simulated patterns, however, did not produce good results because they were found to cross-correlate with other species. At this time more simulated input studies need to be made before a generalized method can be found and applied to different types of microorganisms.

In conclusion, the present preliminary study reveals that the MSF made from a simulated pattern may be useful in recognizing biological species with considerably less size and orientation dependence, which, in turn, helps reduce the total number of filters required for the analysis.

The authors thank G. Indebetouw, S. K. Case for helpful discussion, and P. Pryfogle for assistance in preparation of the biological input.

This research was supported by the National Science Foundation/ASRA division grant ENV72-03581-A05.

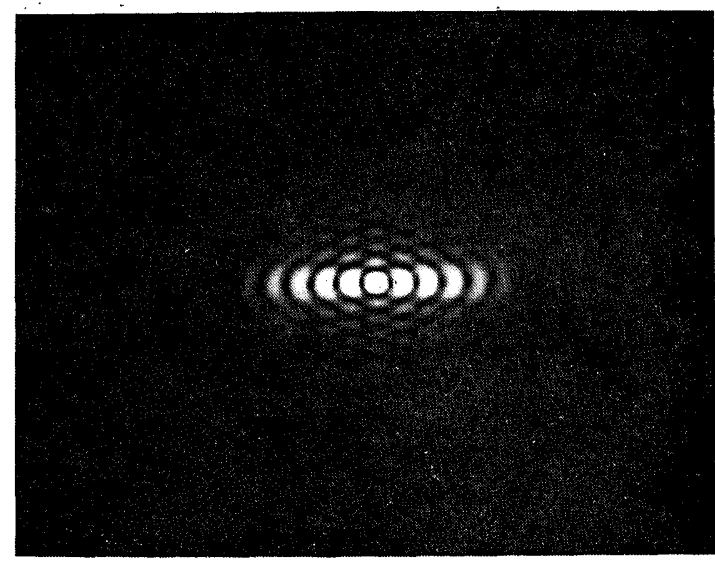

(a)

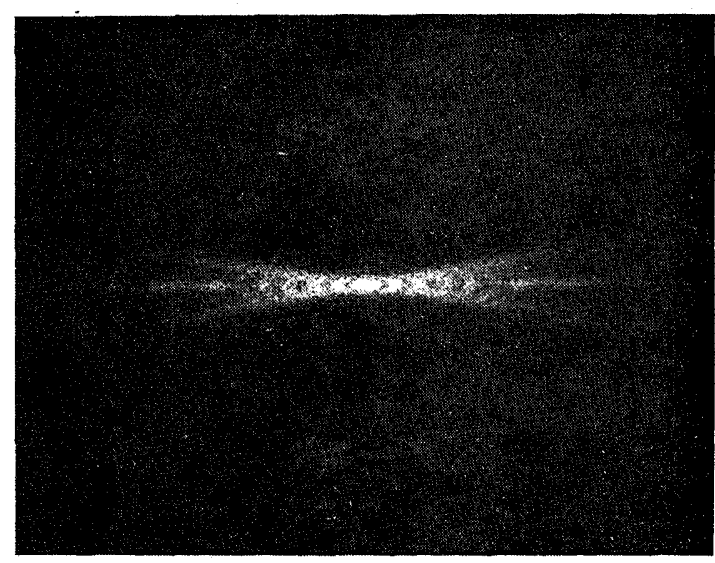

(b)

Fig. 6. (a) The power spectrum of the horseshoe input; (b) the average power spectrum of 20 randomly distributed diatoms of the type shown in Fig. 1(a) averaged for size but having the same orientation. 

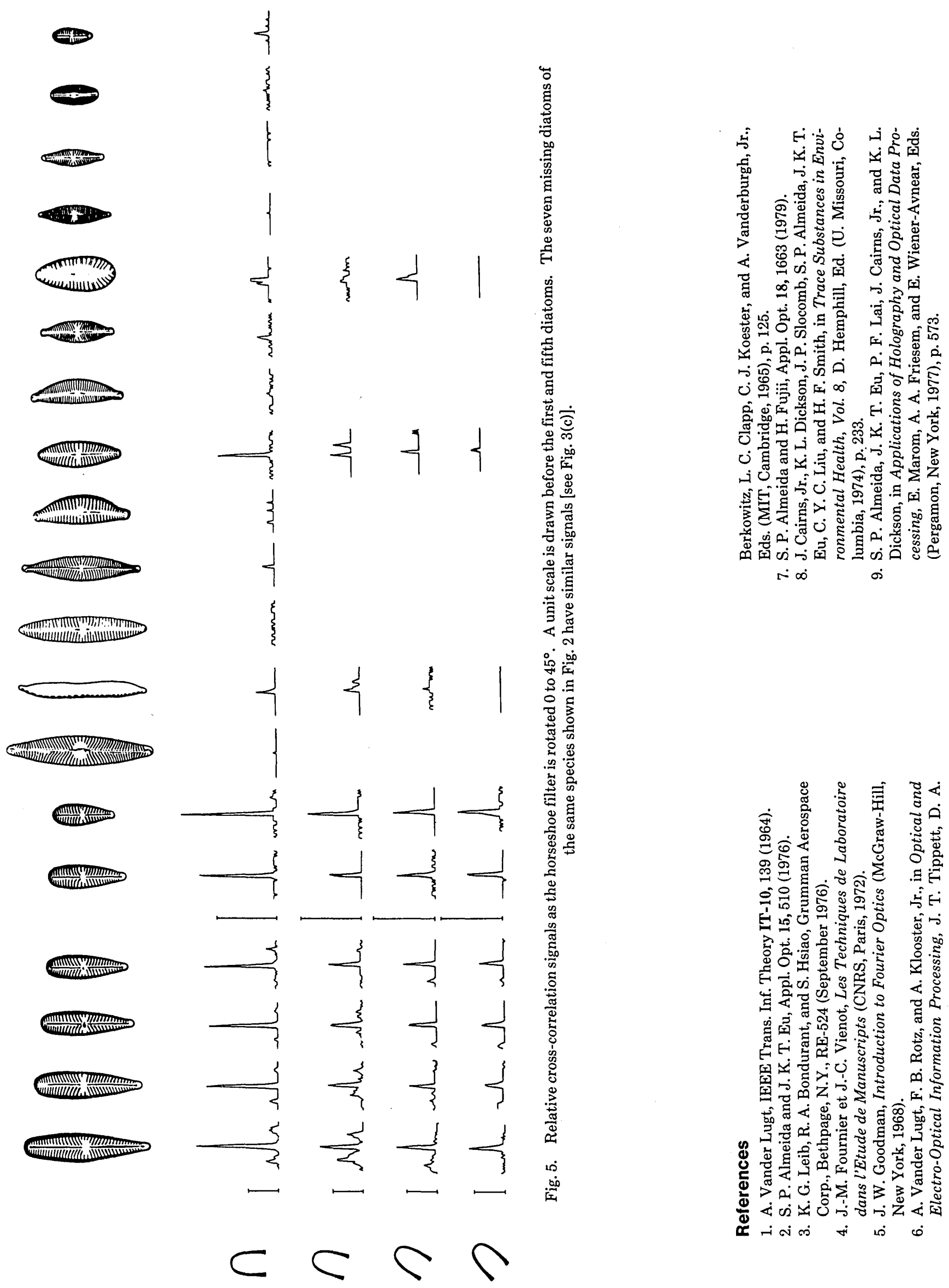